\title{
REDUCTION OF THE SINGULARITIES OF FOLIATIONS AND APPLICATIONS
}

\author{
FELIPE CANO \\ Dpto. Algebra, Geometría y Topología, Fac. Ciencias, Univ. Valladolid \\ E-47005 Valladolid, Spain \\ E-mail:fcano@cpd.uva.es
}

Dedicated to Stanistaw Eojasiewicz on his $70^{\text {th }}$ birthday

1. Introduction. A singular foliation of codimension one is locally given by an integrable 1-form. In the non-singular points the (regular) Frobenius' Theorem provides the description of the pieces of leaves for the foliation. By repeated blowings up of the ambient space, the singularities of the foliation may be transformed into simple ones, that are persistent under new blowings up.

Here we will explain the precise statement of the reduction of singularities for codimension one foliations and the known results. Also we will explain how these results and ideas apply to solve two problems: the existence of integral hypersurface and the description of the frontier of a pfaffian hypersurface. The first one is stated in the complex case; the second one is a problem over the real numbers in the spirit of the theory of subanalytic sets.

2. Singular foliations of codimension one. An ambient space $N$ is a non-singular analytic manifold either over the real numbers $\mathbf{R}$ or over the complex numbers $\mathbf{C}$. A singular foliation $\mathcal{F}$ of codimension one over $N$ is locally given by a 1 -form

$$
\omega=\sum_{i=0}^{n} a_{i}(x) d x_{i}
$$

which is integrable (that is $\omega \wedge d \omega=0$ ) and such that the coefficients $a_{i}$ have no common factor. More precisely, we interpret the foliation $\mathcal{F}$ as the differential equation $\omega=0$ and thus we define it as the sub- $\mathcal{O}_{N}$-module of the cotangent sheaf $\mathcal{F} \subset \Omega_{N}$ locally generated by the 1 -form $\omega$. Up to multiply by a convenient factor, a foliation is locally uniquely determined by any non-null integrable meromorphic 1-form.

Research partially supported by DGICYT and TMR-ERBFMRX-CT96-0040.

1991 Mathematics Subject Classification: Primary 32xxx; Secondary 34xxx.

Received by the editors: October 1, 1997.

The paper is in final form and no version of it will be published elsewhere. 
The singular locus $\operatorname{Sing} \mathcal{F}$ of the foliation $\mathcal{F}$ is locally defined by

$$
\text { Sing } \mathcal{F}=\{P \in N ; \omega(P)=0\} .
$$

It is a closed analytic subset of codimension at least two.

Consider a germ of hypersurface $H$ at a point $P$ in $N$, given by a local equation $h=0$. We say that $H$ is an integral hypersurface of $\mathcal{F}$ if and only if

$$
\omega \wedge d h=h \eta
$$

for a certain analytic 2-form $\eta$. This means that the tangent space of $H$ is the kernel of the cotangent vector $\omega(Q)$ in each point of $H$, outside the singularities (of $H$ and $\mathcal{F}$ ). A germ of function $f$ gives a first integral of $\mathcal{F}$ if

$$
\omega \wedge d f=0 .
$$

That is, each level $f=$ const. is an integral hypersurface. Malgrange Singular Frobenius [30] assures the existence of a first integral if the codimension of the singular locus Sing $\mathcal{F}$ is at least three. In particular, we obtain in this way the pieces of the leaves for the nonsingular foliation induced over $N-\operatorname{Sing} \mathcal{F}$.

Recall that taking local coordinates $\left(x_{1}, \ldots, x_{n}\right)$, a germ $f \in \mathcal{O}_{N, P}$ of function at $P$ is represented by a convergent power series

$$
f=\sum f_{i_{1} \ldots i_{n}} x_{1}^{i_{1}} \ldots x_{n}^{i_{n}} .
$$

Consider a (reduced) formal series $\hat{f}=\sum \hat{f}_{i_{1} \ldots i_{n}} x_{1}^{i_{1}} \ldots x_{n}^{i_{n}}$. We say that $\hat{f}=0$ is a formal integral hypersurface if $\omega \wedge d \hat{f}=\hat{f} \hat{\eta}$ for a certain (formal) 2-form $\hat{\eta}$. For example, the Euler equation

$$
(y-x) d x-x^{2} d y=0
$$

has exactly one (convergent) integral curve given by $x=0$ and one (no convergent) formal integral curve, given by $y=\sum_{m \geq 0} m ! x^{m+1}$.

Let $g: N^{\prime} \rightarrow N$ be a morphism of ambient spaces. We define locally the transform $g^{-1} \mathcal{F}$ of $\mathcal{F}$ by $g$ by considering the analytic integrable 1 -form $g^{*} \omega$. In general we have to divide $g^{*} \omega$ by the local greatest common divisor of the coefficients in order to obtain a generator of $g^{-1} \mathcal{F}$. Note also that we do not exclude the degenerate case $g^{-1} \mathcal{F} \equiv 0$, it corresponds to a morphism $g$ tangent to $\mathcal{F}$. Anyway, this never occurs when $g$ is a blowing up, because it induces an isomorphism between dense open sets.

3. Reduction of singularities in dimension two. The aim of the reduction of singularities of $\mathcal{F}$ is to get a foliation with simpler singularities after a finite sequence of blowings up of the ambient space. A first remark is that we cannot expect to destroy all the singularities of $\mathcal{F}$ by this procedure. For instance, if we blow up the origin of $\left(\mathbf{C}^{2}, 0\right)$, the transform of the non-singular foliation $d x=0$ is locally given by $y d x+x d y=0$ and, repeating the blowings up, we always get singularities of the type $p y d x+q x d y=0$. This kind of singularities correspond to the fact of getting normal crossings for the total transform in the problem of reduction of singularities for the case of varieties.

Our first task is then to identify the kind of singularities that we will get as final singularities by means of the blowing up procedure. We shall call them simple singularities.

Write in dimension two

$$
\omega=a(x, y) d x+b(x, y) d y
$$


Then the foliation is also given locally by the vector field $\mathcal{X}=b(x, y) \partial / \partial x-a(x, y) \partial / \partial y$. One can try to define the simple singularities by the fact that we have multiplicity one (that is the minimum of the multiplicities of $a(x, y)$ and $b(x, y)$ is one). But first this is not stable under blowing up and second this kind of foliations can correspond to singular functions without normal crossings. For instance, take the cusp

$$
\omega=d\left(y^{2}-x^{5}\right)=2 y d y-5 x^{4} d x .
$$

Blowing up the origin, we get a point where the transform of the foliation is given by $2 x y d y+\left(2 y^{2}-x^{4}\right) d x$, which has multiplicity two. The differential of a singular function having normal crossings gives in two variables a vector field whose linear part is nonnilpotent (in fact it has two distinct eigenvalues with negative rational ratio). The fact that the linear part of $\mathcal{X}$ is non-nilpotent is stable under blowing up (for the singular points). This property corresponds to the singularities that we are going to call pre-simple singularities. Anyway consider the foliation given by $d\left(x^{p} / y^{q}\right)=0$, that is

$$
\omega=p y d x-q x d y .
$$

It has infinitely many integral curves that are singular cusps. After blowing up we get two singular points of the type $(p-q) y d x-q x d y$ and $p y d x-(q-p) x d y$. In finitely many steps, we arrive to the radial foliation $y d x-x d y=0$. Blowing up this one, the singularity disappears and the exceptional divisor of the blowing up becomes transversal to the transformed foliation (dicritical situation). The above phenomenon occurs only because of the ratio of the eigenvalues is rational positive. Thus,

In dimension two a singularity of foliation is called to be simple if and only if the linear part of the vector field $\mathcal{X}$ has two distinct eigenvalues $\lambda \neq \mu \neq 0$ such that the quotient $\lambda / \mu$ is not a positive rational number.

Remark that one of the eigenvalues can be zero, is this case we say that we have a saddle node, for example, the Euler equation. The simple singularities are stable under blowing up and non-dicritical in the sense that the exceptional divisor after a blowing up is always an integral curve. But they can completely disappear after blowing up because of a reason of algebraic nature, take for instance the real center given by $d\left(x^{2}+y^{2}\right)=0$. The corresponding linear part has conjugate non-real eigenvalues and after blowing up we get a foliation "locally parallel" to the exceptional divisor. If we are placed over a non-dicritical normal crossings divisor (the irreducible components are integral curves) containing $x=0$ this situation never occurs, since we can write

$$
\omega=x\left(a(x, y) \frac{d x}{x}+\tilde{b}(x, y) d y\right) .
$$

Then the two eigenvalues are necessarily in the base field (since $n-1$ of them are so). That is why we take the following definition

Definition 1. We say that a singularity of the foliation $\mathcal{F}$ over an ambient space of dimension two is simple adapted to a normal crossings divisor $E$ if and only if it is simple, there is at least one irreducible component of $E$ passing through it and all the irreducible components of $E$ are non-dicritical for $\mathcal{F}$ (integral hypersurfaces).

Blowing up a simple singularity adapted to $E$ produces exactly two simple singularities adapted to the total transform of $E$; the strict transform of each irreducible component of $E$ passes through one of these singular points. If $E$ has two irreducible components 
(call this a coin) we see in this way that they are the only integral curves of the foliation: otherwise the strict transform of a third integral curve will pass through a not coin after finitely many blowings up, creating an impossible third singularity. Assume that $E$ has only one irreducible component $x=0$. Blowing up indefinitely, we find a sequence of infinitely near points corresponding to singularities which are not coins: they determine a non-singular formal curve $\Gamma$ transversal to $x=0$. This curve $\Gamma$ is a formal integral curve and the same argument as above shows that $E$ and $\Gamma$ are the only formal integral curves of $\mathcal{F}$. Moreover assume that we can write

$$
\omega=x\left(\frac{d x}{x}+(-\lambda+\phi(x, y)) d y\right)
$$

where $\phi(0,0)=0$ and $\lambda \notin \mathbf{Q}_{+}$. Then Briot and Bouquet theorem [3] states that the curve $\Gamma$ is in fact a convergent curve. In this case we say that the eigenspace (of the linear part of the vector field $\mathcal{X}$ ) transverse to the exceptional divisor is strong.

Let us look at the dicritical components that we can produce by blowing up. Let $\nu$ be the minimum of the orders at the point $P$ of the coefficients $a(x, y)$ and $b(x, y)$ of $\omega$. After one blowing up, the exceptional divisor is generically transversal (dicritical) to the transform of the foliation if and only if

$$
\nu_{P}(\omega(x \partial / \partial x+y \partial / \partial y)=x a(x, y)+y b(x, y)) \geq \nu+2 .
$$

Thus, creating dicritical components by blowing up is possible. Actually (but only in dimension two) it corresponds to the fact that $\mathcal{F}$ has infinitely many integral curves (project by the sequence of blowings up the integral transversal curves in the generic points of the dicritical component). We want these dicritical components to have normal crossings with the foliation after reduction of singularities. The precise definition is

Definition 2. Let $D$ be a normal crossings divisor over an ambient space $N$ of dimension two and fix a point $P \in N$. We say that foliation $\mathcal{F}$ has normal crossings with $D$ at the point $P$ if one of the following properties occurs:

1. The point $P$ is a singular point of $\mathcal{F}$ and it is simple adapted to $D$. In particular, all the irreducible components of $D$ through $P$ are non-dicritical for $\mathcal{F}$.

2. The point $P$ is non-singular for $\mathcal{F}$ and there are local coordinates $(x, y)$ at $P$ such that $\mathcal{F}$ is given by $d x=0$ and $D \subset(x y=0)$.

Now, we can state the result of reduction of singularities in dimension two that is essentially due to Seidenberg [35].

Theorem 3 (Reduction of singularities in dimension two). Consider a singular foliation $\mathcal{F}$ over an ambient space $N$ of dimension two and fix a point $P \in N$. There is a finite sequence of blowings up of points over the point $P$

$$
N \stackrel{\pi_{1}}{\longleftarrow} N_{1} \stackrel{\pi_{2}}{\longleftarrow} N_{2} \ldots \stackrel{\pi_{k}}{\longleftarrow} N_{k}=N^{\prime} .
$$

Such that the transform $\mathcal{F}^{\prime}$ of $\mathcal{F}$ in $N^{\prime}$ has normal crossings with the total exceptional divisor $D^{\prime}$ at each point of $D^{\prime}$. In particular, any singularity of $\mathcal{F}^{\prime}$ over $D^{\prime}$ is a simple singularity for $\mathcal{F}^{\prime}$ adapted to $D^{\prime}$.

4. Pre-simple singularities. The proofs of the propositions in this section are in [9] in the three-dimensional non-dicritical case and in [8] in the general case, in any ambient dimension. 
In order to define simple singularities (and first pre-simple ones) in higher dimension, we need the idea of dimensional type. Given a point $P$ of the ambient space $N$, the dimensional type $\tau(\mathcal{F}, P)$ of the foliation $\mathcal{F}$ at the point $P$ is the codimension in $T_{P} N$ of the vector space

$$
\mathcal{D}(\omega)(P)=\{\mathcal{X}(P) ; \omega(\mathcal{X})=0\}
$$

where $\mathcal{X}$ denotes a germ of vector field at $P$. Put $t=\tau(\mathcal{F}, P)$. Then, up to selecting good coordinates and a generator $\omega$ of $\mathcal{F}$, we can write $\omega$ only in the first $t$ coordinates

$$
\omega=\sum_{i=0}^{t} a_{i}\left(x_{1}, \ldots, x_{t}\right) d x_{i} .
$$

Let $E$ be a normal crossings divisor of $N$ such that all the irreducible components of $E$ are non-dicritical (integral hypersurfaces). Denote by $e=e(E, P)$ the number of irreducible components of $E$ through $P$. It is evident that $e \leq t$. In particular we can take coordinates $\left(x_{1}, \ldots, x_{n}\right)$ at $P$ and a subset $A \subset\{1, \ldots, t\}$ such that

$$
E=\left(\prod_{i \in A} x_{i}=0\right)
$$

and then $\omega$ is written down in the following way

$$
\omega=\left(\prod_{i \in A} x_{i}\right)\left[\sum_{i \in A} b_{i}\left(x_{1}, \ldots, x_{t}\right) \frac{d x_{i}}{x_{i}}+\sum_{i \in\{1, \ldots, t\}-A} b_{i}\left(x_{1}, \ldots, x_{t}\right) d x_{i}\right]
$$

where the coefficients $b_{i}$ are germs of functions without common factor. This presentation of $\omega$ is very convenient for the control of the singularities in the problem of reduction. Also it will serve to us to define simple singularities.

The adapted order $\nu(\mathcal{F}, E ; P)$ is defined to be the minimum of the orders at $P$ of the coefficients $b_{i}$; it is obviously an upper semicontinuous invariant. The adapted multiplicity $\mu(\mathcal{F}, E ; P)$ is by definition the order at $P$ of the ideal generated by

$$
\left\{b_{i}\right\}_{i \in A} \cup\left\{x_{j} b_{i}\right\}_{i \notin A, j \in\{1, \ldots, n\}} .
$$

Note that $\nu(\mathcal{F}, E ; P) \leq \mu(\mathcal{F}, E ; P) \leq \nu(\mathcal{F}, E ; P)+1$.

Definition 4. We say that a point $P \in \operatorname{Sing} \mathcal{F}$ is a pre-simple singularity for $\mathcal{F}$ adapted to $E$ if and only if one of the following properties holds:

1. $\nu(\mathcal{F}, E ; P)=0$;

2. $\nu(\mathcal{F}, E ; P)=\mu(\mathcal{F}, E ; P)=1$ and there is a coefficient $b_{i}$ with $i \in A$ such that the linear part of $b_{i}$ does not depend only on $\left\{x_{i} ; i \in A\right\}$.

Denote by $\operatorname{Sing}^{*}(\mathcal{F}, E)$ the set of singular points $P \in \operatorname{Sing} \mathcal{F}$ that are not pre-simple singularities for $\mathcal{F}$ adapted to $E$.

Proposition 5 (Horizontal stability of pre-simple singularities). The non-pre-simple singularities $\operatorname{Sing}^{*}(\mathcal{F}, E)$ form a closed analytic set of the ambient space $N$.

In order to assure the vertical stability (persistency under permissible blowings up) we need the following results that also gives a partial geometric description of the pre-simple singularities. 
Proposition 6 .

a) Consider a pre-simple singularity $P \in \operatorname{Sing} \mathcal{F}-\operatorname{Sing}^{*}(\mathcal{F}, E)$. Then the dimensional type satisfies

$$
\begin{gathered}
t=\tau(\mathcal{F}, P)=\nu(\mathcal{F}, \emptyset ; P)+1 \\
e=e(E, P)-1 \leq t \leq e .
\end{gathered}
$$

b) Assume that $\nu(\mathcal{F}, E ; P)=\mu(\mathcal{F}, E ; P)=1$ (note that then $t=e-1$ ). Take coordinates $\left(x_{1}, \ldots, x_{n}\right)$ such that $E=\left(\prod_{i=1}^{t-1} x_{i}=0\right)$, write a generator $\omega$ of $\mathcal{F}$ as above and denote by $B_{i}$ the linear part of the coefficient $b_{i}$. Then

1. There is exactly one formal hypersurface $\hat{H}$ different from the irreducible components of $E$ such that $\hat{H}$ is an integral hypersurface for $\mathcal{F}$ and $E \cup \hat{H}$ has normal crossings. Moreover, let $x_{t}=\phi\left(x_{1}, \ldots, x_{t-1}\right)$ be an equation for $\hat{H}$ and write for any index $k$

$$
\phi\left(x_{1}, \ldots, x_{t-1}\right)=\sum_{s=1}^{\infty} \phi_{s}^{(k)}\left(x_{1}, \ldots, x_{k-1}, x_{k+1}, \ldots, x_{t-1}\right) x_{k}^{s}
$$

then the $\phi_{s}^{(k)}$ are convergent series in $t-2$ variables with a common domain of convergence.

2. The linear parts $B_{i}, i \in A$, are not resonant in the sense that there is no function $\Phi: A \mapsto \mathbf{Z}_{+}$such that $\sum_{i \in A} \Phi(i) B_{i}=0$.

For the construction of $\hat{H}$, first we find step by step in a unique way a formal coordinate change $\hat{x_{t}}=x_{t}-\phi\left(x_{1}, \ldots, x_{t-1}\right)$ such that $\hat{x_{t}}$ divides the first coefficient of $\omega$. That is,

$$
\omega=\hat{x}_{t} \frac{d x_{1}}{x_{1}}+\sum_{s=2}^{t-1} b_{s} \frac{d x_{s}}{x_{s}}+b_{t} d x_{t}
$$

The fact that $\phi$ is only transversely formal is easily derived from this construction. Now, the integrability condition $\omega \wedge d \omega=0$ implies that $\hat{x_{t}}$ divides $b_{s}$, for $s=2, \ldots, t-1$. The non-resonance is now a consequence of the integrability condition and of the fact that $b_{t}$ has order at least one.

The general stability of the control invariants under permissible blowings up (developed in [7] for the non-dicritical case and in [8] for the general case) states that the adapted order is always stable and the adapted multiplicity is stable if we are not radially dicritical (a resonant case with $\Phi(i)=1$ for all $i \in A$ ). Hence we deduce the following result of persistency for pre-simple singularities.

Proposition 7 (Vertical stability of pre-simple singularities). Assume that we have only pre-simple singularities, that is $\operatorname{Sing}^{*}(\mathcal{F}, E)=\emptyset$. Let $Y \subset \operatorname{Sing} \mathcal{F}$ be a closed nonsingular subspace of the ambient space $N$ having normal crossings with $E$ and consider the blowing up $\pi: N^{\prime} \rightarrow N$ of $N$ with center $Y$. Denote by $E^{\prime}$ the set of non-dicritical components of $\pi^{-1}(E \cup Y)$ for the transform $\mathcal{F}^{\prime}$ of $\mathcal{F}$ by $\pi$. Then

$$
\operatorname{Sing}^{*}\left(\mathcal{F}^{\prime}, E^{\prime}\right)=\emptyset \text {. }
$$

That is, all the singularities of $\mathcal{F}^{\prime}$ are pre-simple singularities adapted to $E^{\prime}$.

Remark 8 . The definition of permissible center that we use in $[7,9,8]$ is more specific that the one suggested in the above proposition, but they coincide in the case we have only pre-simple singularities. Actually, we use two definitions, both of them inspired in the equimultiplicity property used as a base by Hironaka for his normal flatness condition [17]. 
The weak condition, that we call permissible center is horizontally stable and is useful for the control of the generic points; the strong condition, that we call appropriate center is not horizontally stable and we use it for the control of the bad points. In the nondicritical case there is a horizontal semicontinuity of the appropriate center condition [7], that simplifies very much the strategy for the reduction of singularities; this result is not true in the general case and it is necessary to solve first the bad points with appropriate centers and second the generic points with only permissible centers [8].

5. Getting pre-simple singularities. The first task in the reduction of the singularities is to eliminate the set $\operatorname{Sing}^{*}(\mathcal{F}, E)$ by means of permissible blowings up of the ambient space. Then, consider the following statement:

Statement 9 (Reduction to pre-simple singularities). Let $\mathcal{F}$ be a singular foliation of codimension one over an ambient space $N$ of dimension $\operatorname{dim} N=n$. Assume that $N$ is a germ around a compact analytic subset $C \subset N$ (call it the core); two important examples are $C=P$ and $C=N$. Then there is a finite sequence of permissible blowings up (of germs around $C$ )

$$
N \stackrel{\pi_{1}}{\longleftarrow} N_{1} \stackrel{\pi_{2}}{\longleftarrow} N_{2} \ldots \stackrel{\pi_{k}}{\longleftarrow} N_{k}=N^{\prime}
$$

such that $\operatorname{Sing}^{*}\left(\mathcal{F}^{\prime}, E^{\prime}\right)=\emptyset$, where $\mathcal{F}^{\prime}$ is the transform of $\mathcal{F}$ in $N^{\prime}$ and $E^{\prime}$ is the divisor composed of the non-dicritical components of the total exceptional divisor in $N^{\prime}$.

This statement is still a conjecture if $n \geq 4$. If $n=2$, its proof is contained in Seidenberg's paper [35]. The proof in the case $n=3$ is done in [7] for the non-dicritical case and in [8] without this assumption. We say that the foliation is non-dicritical if for any sequence of permissible blowings up the last exceptional divisor is always nondicritical. In $[5,6]$ we give other characterizations of this property; we shall return to it in the section about the existence of integral hypersurfaces. The reduction to the pre-simple singularities concentrates the main difficulty in the problem of reduction of singularities of foliations. Although the proof is out of the scope of this expository paper, let us say at least what are the main ideas in the known case of dimension three.

First, let us remark that the reduction to the pre-simple singularities of a foliation given by $\omega=d f$ is the same one as the reduction of the singularities of the analytic set $f=0$. In fact in $[18,1,2]$ it is proved that after finitely many blowings up the total transform of $f$ is locally a monomial compatible with the exceptional divisor (without dicritical components) and with at most one irreducible component outside of the divisor: this corresponds to a pre-simple singularity. Conversely, if we have a pre-simple singularity of the form $\omega=d f$ we are dealing locally with a monomial of the above type. Thus, the difference between pre-simple and simple singularities, to be seen later, is outside of the example $\omega=d f$. Anyway, the reduction to the pre-simple singularities is quite more complicated than the corresponding problem for a hypersurface, mainly in the dicritical case. Two key difficulties appear, first there is no a clear substitute of the Hilbert-Samuel function used by Hironaka and second we have to deal with resonance phenomena that do not exist in the problem of varieties in characteristic zero (in fact we think in parallelisms for the characteristic $p$ cases; see $[15,16,14,38])$.

We construct a set of invariants that will correspond, in a non-complete way, to the idea of the Hilbert-Samuel function. This family works in any dimension and is divided into invariants of transversality and invariants of resonance. The main invariants 
of transversality are the adapted order, say $r$, the adapted multiplicity, say $m$, and the directrix, call it $\operatorname{Dir}(\mathcal{F}, E ; P)$. The directrix is our version of the strict tangent space of Hironaka. Given an homogeneous polynomial $F\left(X_{1}, \ldots, X_{n}\right)$ denote by $\operatorname{Dir}(F)$ the maximum linear subspace that leaves the cone $F=0$ invariant by translation. Taking the above local adapted notation with coordinates $b_{i}$ for the form $\omega$, we define

$$
\operatorname{Dir}(\mathcal{F}, E ; P)= \begin{cases}\bigcap_{i \in A} \operatorname{Dir}\left(\operatorname{In}^{r} b_{i}\right) & \text { if } m=r \\ \bigcap_{i \notin A} \operatorname{Dir}\left(\operatorname{In}^{r} b_{i}\right) & \text { if } m=r+1\end{cases}
$$

where $\operatorname{In}^{r} b_{i}$ means the initial part of degree $r$ of the coefficient $b_{i}$. The directrix has a good behavior under appropriate blowings up: it determines the possible situation of the points in the exceptional divisor for which the main invariants $r$ and $m$ do not drop, except perhaps in the radially dicritical case. Moreover, another family of transversality invariants is derived from the directrix; we extract from them the invariant $d$, of transversal to $E$ codimension of the directrix. It measures how many independent linear forms, modulo $X_{i}$ for $i \in A$, appear in the equations of the directrix. The codimension of the directrix is not necessarily stable under blowings up, but this invariant $d$ is either increasing or stable (always outside of the radially dicritical case). The case $d=0$ is called hidden directrix; it is a priori the most difficult case, but we can show that if this case is stable we are always over the strict transform of certain components of the exceptional divisor that we call contact components and essential components and hence we get a maximal contact situation.

The resonance invariant $\operatorname{Rs}(\mathcal{F}, E ; P)$ takes three possible logical values $0,1,2$. The value 1 corresponds to the radially dicritical case, the value 2 to the other resonant cases with $m=r$ and $\operatorname{Rs}(\mathcal{F}, E ; P)=0$ otherwise. We modify the invariant $m$ to get the invariant $m^{*}$ defined by $m^{*}=m$ if $\operatorname{Rs}(\mathcal{F}, E ; P)=0$ and $m^{*}=m+1$ otherwise. The pair $\left(r, m^{*}\right)$ is always stable under appropriate blowing up and will act as the Hilbert-Samuel function in our problem of reduction of singularities for the so called bad points.

Once this collection of invariants and of general definitions is established, we use them to proof the existence of a reduction to pre-simple singularities in dimension three. This problem has two parts; first a global criteria of blowing up that allows us to choose globally the next center of blowing up and second a local control of this possibly infinite sequence of global blowings up that implies that the sequence is not infinite. As we have already said, we will separate the generic behavior from the bad one; this is in fact the base of the global criteria and for this we need the idea of generic equireduction.

Definition 10. Consider a point $P \in \operatorname{Sing}^{*}(\mathcal{F}, E)$. We say that $P$ is a good point, or equivalently a point over which we have generic equireduction if and only if there is a neighborhood $U$ of $P$ and a finite sequence of blowings up

$$
U \stackrel{\pi_{1}}{\longleftarrow} U_{1} \stackrel{\pi_{2}}{\longleftarrow} U_{2} \ldots \stackrel{\pi_{k}}{\longleftarrow} U_{k}
$$

such that $\operatorname{Sing}^{*}\left(\mathcal{F}_{k}, E_{k}\right)=\emptyset$ and for any index $j=1, \ldots, k$ the $\operatorname{locus}_{\operatorname{Sing}}{ }^{*}\left(\mathcal{F}_{j-1}, E_{j-1}\right)$ is non-singular, of codimension two, etale over $\operatorname{Sing}^{*}(\mathcal{F}, E)$ and it is the center of the next blowing up $\pi_{j}$.

Call bad points the set $\operatorname{Bd}(\mathcal{F}, E)$ of points in $\operatorname{Sing}^{*}(\mathcal{F}, E)$ which are not good points. In [10] there is a proof of the following result (in any ambient dimension).

Proposition 11 (Generic equireduction). The set $\operatorname{Bd}(\mathcal{F}, E)$ is a closed analytic set of $N$ of codimension at least three. 
In particular, if $n=3$ the set of bad points is a set of isolated points. The idea is first to eliminate the bad points. Once this done the good points will disappear by a finite number of canonical global blowings up in view of the generic equireduction. Thus the global strategy of blowings up looks prior to the bad points having maximum invariant $\left(r, m^{*}\right)$ (a finite number of points). We do not do the details here (see [7] in the nondicritical case and [8] in the general case) but let us remark the main properties of this global strategy:

1. If there is one bad point having the initial maximum Samuel invariant $\left(r, m^{*}\right)$, then we can always choose a global center accordingly with the global criteria. This center is always appropriate at the bad points and permissible elsewhere.

2. After finitely many global blowings up following this strategy, we always modify at least one bad point (that is there is at least one bad point included in the corresponding center).

3. Locally at one bad point we priorize it as center when it is a radially dicritical corner (three components of the divisor). Otherwise, we priorize the appropriate centers of biggest dimension.

Let us do the global criteria to work. This produces a sequence of global blowings up. If it stops, we get bad points with only smaller invariant $\left(r, m^{*}\right)$. By induction we can eliminate the bad points and by generic equireduction we obtain the desired reduction to pre-simple singularities. Thus we have only to consider the case where the sequence does not stop and to prove that this is not possible. If we get an infinite sequence, we can specialize it to an infinite sequence of local blowings up

$$
\mathcal{B} \equiv\left\{\begin{array}{l}
W \stackrel{\pi_{1}}{\longleftarrow} W_{1} \stackrel{\pi_{2}}{\longleftarrow} W_{2} \ldots \stackrel{\pi_{j}}{\longleftarrow} W_{j} \stackrel{\pi_{j+1}}{\longleftarrow} \ldots \\
P_{0} \longleftarrow P_{1} \longleftarrow P_{2} \ldots \longleftarrow P_{j} \longleftarrow \ldots
\end{array}\right.
$$

where $W_{j}$ is a small neighborhood of $P_{j}$ and $P_{j+1}$ maps to $P_{j}$. Call this sequence $\mathcal{B}$ of local blowings up an infinite bamboo of infinitely near points (it can also be seen as a star in the sense of Hironaka [19]). This infinite bamboo $\mathcal{B}$ has the property that at each $P_{j}$ the invariant $\left(r, m^{*}\right)$ is the same one. Moreover the centers of the local blowings up, being specialization of the centers given by the global criteria, satisfy to the local conditions of the global criteria. Now we have to prove that this infinite bamboo cannot exist and this ends the proof of the reduction to pre-simple singularities.

Assume by contradiction that $\mathcal{B}$ exists. We separate two main cases: the $m$-stable case and the $m$-unstable case. The $m$-stable case corresponds to the case in which the adapted multiplicity $m$ is the same one at each level $P_{j}$. The $m$-unstable case corresponds to the fact that $m=r$ and $m=r+1$ occurs infinitely many times (in particular $m^{*}=r+1$ and the steps with $m=r$ are resonant ones).

For the control of the $m$-stable case, we consider first the situation where we have a non-singular surface $H$ having maximal contact with the bamboo $\mathcal{B}$. This means that all the centers of the blowings up (and hence the points $P_{j}$ ) are contained in the corresponding strict transforms of $H$. In that case, we can develop a concept of differential idealistic exponent in the same spirit of [20]; we project in this way our reduction problem to related problem in dimension two over the ambient space $H$ whose solution is easily done. Now we need to get this maximal contact situation. For this we essentially use all our collection of invariants; they are stable under appropriate blowing up and so we can assume that they are fixed along the bamboo $\mathcal{B}$. This helps us to identify maximal 
contact surfaces. Let us only remark here that we need in some (quite transversal) cases to add to our list of invariants some ones obtained directly from a characteristic polygon that we draw by selecting coordinates very well prepared with respect to the bamboo $\mathcal{B}$; this characteristic polygon is inspired in [21, 22].

Finally, the $m$-unstable case is treated by identifying several hierarchized situations or levels (around ten of them, but not complicated to define); each one is $m$-stable, hence we have to change of level infinitely many times. But each time we change, we go to a lower level. This ends the proof.

6. Simple singularities. The best way to define simple singularities is to establish formal normal forms for the pre-simple singularities. This also gives a finer description of the pre-simple singularities. The formal normal forms are directly derived from the jordanization of commuting formal vector fields and from the formal normal form for a 1-form in one variable. The main ideas are collected in Martinet's Bourbaki [31], the formal normal forms in dimension three for the non-dicritical case are in [9] and in the general case in [8] (but there is no essential difference between the three-dimensional case and the general case).

Take a pre-simple singularity $P \in \operatorname{Sing} \mathcal{F}$ adapted to $E$. Assume without loss of generality that the dimensional type of $\mathcal{F}$ at $P$ is $n=\operatorname{dim} N$. Then we know that $E$ has either $n-1$ or $n$ irreducible components through $P$. Taking account of the results in the section about pre-simple singularities, we easily find $n-1$ germs of formal vector fields of the type

$$
\mathcal{X}_{j}=x_{j} \frac{\partial}{\partial x_{j}}-c_{j}\left(x_{1}, \ldots, x_{n}\right) \frac{\partial}{\partial x_{n}},
$$

$j=1, \ldots, n-1$, where $c_{j}(0)=0$, that are tangent to the foliation and that commutes (this is directly given by the integrability condition). Then we can decompose the vector fields in semisimple and nilpotent parts as

$$
\mathcal{X}_{j}=\mathcal{S}_{j}+\mathcal{N}_{j}
$$

in such a way that $\left[\mathcal{S}_{j}, \mathcal{S}_{s}\right]=0$ and $\left[\mathcal{S}_{j}, \mathcal{N}_{s}\right]=0$ for any indices $j, s$. Moreover there is a formal coordinate system $\left(x_{1}, \ldots, x_{n}\right)$ such that the semisimple parts have a diagonal expression

$$
\mathcal{S}_{j}=x_{j} \frac{\partial}{\partial x_{j}}-\lambda_{j} \frac{\partial}{\partial x_{n}} .
$$

The commuting properties imply now strong conditions over the expression of the nilpotent parts in the coordinates $\left(x_{1}, \ldots, x_{n}\right)$ (for instance, if the eigenvalues $\lambda_{j}$ are independent over $\mathbf{Z}$, necessarily the nilpotent parts are zero). We can reconstruct a generator $\omega$ of the foliation from the $n-1$ vector fields above. The result is summarized in the following proposition.

Proposition 12 (Jordanization of a pre-simple singularity). Let $P \in \operatorname{Sing} \mathcal{F}$ be a pre-simple singularity adapted to $E$ and put $t=\tau(\mathcal{F}, P)$. Then there is a formal coordinate system $\left(x_{1}, \ldots, x_{t}\right)$ at $P$ such that $E \subset\left(\prod_{i=1}^{t} x_{i}=0\right)$ and a generator $\omega$ of $\mathcal{F}$ is written in one of the following forms: 
A) There are complex numbers $\lambda_{i} \in \mathbf{C}^{*}, i=1, \ldots, t$ (that will be real ones if we work over the real numbers) such that

$$
\omega=\left(\prod_{i=1}^{t} x_{i}\right)\left[\sum_{i=1}^{t} \lambda_{i} \frac{d x_{i}}{x_{i}}\right]
$$

B) There is an integer $k \geq 1$, a k-uple $\left(p_{1}, \ldots, p_{k}\right) \in\left(\mathbf{Z}_{+}\right)^{k}$, complex numbers $\lambda_{i} \in \mathbf{C}$, $i=2, \ldots, t$ with $\lambda_{i} \in \mathbf{C}^{*}$ for $i=k+1, \ldots, t$ and a one variable non-null formal series $\Psi(T)$ with $\Psi(0)=0$ such that

$$
\omega=\left(\prod_{i=1}^{t} x_{i}\right)\left[\sum_{i=1}^{k} p_{i} \frac{d x_{i}}{x_{i}}+\Psi\left(x_{1}^{p_{1}} \ldots x_{k}^{p_{k}}\right) \sum_{i=2}^{t} \lambda_{i} \frac{d x_{i}}{x_{i}}\right] .
$$

C) There is an integer $k \geq 2$, a $(k-1)$-uple $\left(p_{2}, \ldots, p_{k}\right) \in\left(\mathbf{Z}_{+}\right)^{k-1}$ and complex numbers $\lambda_{i} \in \mathbf{C}, i=2, \ldots, t$ with $\lambda_{t} \neq 0$ and $\lambda_{i} \neq 0$ for $i=k+1, \ldots, t$ such that

$$
\omega=\left(\prod_{i=2}^{t} x_{i}\right)\left[d x_{1}-x_{1} \sum_{i=2}^{k} p_{i} \frac{d x_{i}}{x_{i}}+x_{2}^{p_{2}} \ldots x_{k}^{p_{k}} \sum_{i=2}^{t} \lambda_{i} \frac{d x_{i}}{x_{i}}\right] .
$$

Remark 13. The case B is given by the pull back of the 1 -form

$$
\frac{1}{\Psi(y)} \frac{d y}{y}+\sum_{i=2}^{t} \lambda_{i} \frac{d x_{i}}{x_{i}}
$$

under the morphism

$$
\left(x_{1}, \ldots, x_{n}\right) \mapsto\left(y, x_{2}, \ldots, x_{n}\right)=\left(x_{1}^{p_{1}} \ldots x_{k}^{p_{k}}, x_{2}, \ldots, x_{n}\right) .
$$

Invoking the normal form of a meromorphic 1-form in one variable, we find a formal coordinate $u$ such that

$$
\frac{1}{\Psi(y)} \frac{d y}{y}=\frac{\delta+\epsilon u^{s}}{u^{s}} \frac{d u}{u}
$$

and so we can write the case B in the formal normal form

$$
\omega=\left(\prod_{i=1}^{t} x_{i}\right)\left[\sum_{i=1}^{k} p_{i} \frac{d x_{i}}{x_{i}}+\frac{x_{1}^{p_{1}} \ldots x_{k}^{p_{k}}}{\delta+\epsilon x_{1}^{p_{1}} \ldots x_{k}^{p_{k}}} \sum_{i=2}^{t} \lambda_{i} \frac{d x_{i}}{x_{i}}\right] .
$$

The above proposition gives us a description of the singular locus Sing $\mathcal{F}$ around a pre-simple singularity. The formal description corresponds by faithful flatness to the convergent one. So the singular locus is composed of non-singular irreducible components of codimension two. Moreover, the natural stratification induced by the non-adapted order $\nu(\mathcal{F}, \emptyset ; P)$ (that is, the minimum of the orders of the coefficients of a holomorphic 1-form that generates $\mathcal{F}$ ) has non-singular strata that are compatible (in the sense of ojasiewicz $[28,29])$ with all the components of the divisor $E$. Since $\nu(\mathcal{F}, \emptyset ; P)=$ $\tau(\mathcal{F}, P)-1$, this stratification is also induced by the dimensional type. Actually, the adherence of the strata corresponding to dimensional type $t$ is a union of non-singular closed analytic subspaces of codimension exactly $t$ in the ambient space. Let $\Gamma$ be one stratum corresponding to dimensional type $t$. We know that $\bar{\Gamma}$ is non-singular and given two points $P, Q \in \Gamma$ the germs of foliation of $\mathcal{F}$ in $P$ and $Q$ are isomorphic to a fixed $(n-t)$-dimensional transversal section of $\mathcal{F}$ in the point $P$ (this generates a kind of holonomy). 
In the above proposition, the types $\mathrm{A}, \mathrm{B}$ and $\mathrm{C}$ are mutually excluding: the type $\mathrm{A}$ can be viewed as a type $\mathrm{B}$ with the invariant $k=0$. Moreover, in the cases $\mathrm{A}$ or $\mathrm{B}$, the vector

$$
\underline{\lambda}=\left(\lambda_{k+1}, \ldots, \lambda_{t}\right) \in\left(\mathbf{C}^{*}\right)^{t-k}
$$

is invariant for the foliation, up to multiplication by a non-null scalar and up to reordering its entries. This allows us to give the following definition:

Definition 14 (Simple singularities). Let $P \in \operatorname{Sing} \mathcal{F}$ be a pre-simple singularity adapted to $E$ and put $t=\tau(\mathcal{F}, P)$. We say that $P$ is a simple singularity adapted to $E$ if and only if we are either in the case $\mathrm{A}$ or $\mathrm{B}$ of the preceding proposition and the vector $\underline{\lambda}$ is not resonant in the sense that

$$
\sum_{j=k+1}^{t} \Phi(j) \lambda_{j} \neq 0
$$

for any $\Phi:\{k+1, \ldots, t\} \rightarrow \mathbf{Z}_{+} \cup\{0\}$ such that $\Phi \neq 0$.

The following proposition summarizes the specific properties for the simple singularities:

Proposition 15. Let $P \in \operatorname{Sing} \mathcal{F}$ be a simple singularity adapted to $E$ and put $t=\tau(\mathcal{F}, P)$. Two cases may occur:

1. $e(E, P)=t$ and then the irreducible components of $E$ are the only integral hypersurfaces of $\mathcal{F}$ at the point $P$. (Call this a simple corner.)

2. $e(E, P)=t-1$ and then there is exactly one transversely formal hypersurface $\hat{H}$ at $P$ such that $E \cup \hat{H}$ has normal crossings and the only integral hypersurfaces of $\mathcal{F}$ at $P$ are $\hat{H}$ and the irreducible components of $E$. (Call this a trace simple singularity.)

If $Y$ is a permissible center for $\mathcal{F}$ adapted to $E$ with $P \in Y$ and $\pi: N^{\prime} \rightarrow N$ is the blowing up with center $Y$, then $\pi$ is non-dicritical and any singular point $P^{\prime}$ over $P$ is a simple singularity. More precisely, if $P$ is a simple corner, we produce only simple corners by the blowing up and if $P$ is a trace simple singularity, the only trace simple singularities that are produced under the blowing up are in the strict transform of $\hat{H}$.

In the case of simple corners, the singular locus and its stratification in terms of the dimensional type is given by the natural stratification induced by the divisor $E$. In the case of trace simple singularities, we get the same result changing $E$ by $E \cup \hat{H}$.

The next step in the problem of reduction of singularities of a singular foliation of codimension one is to eliminate the pre-simple singularities that are not simple singularities. This can be done in any dimension [8] and the precise statement is the following one:

THEOREM 16 (Reduction to simple singularities). Let $\mathcal{F}$ be a singular foliation of codimension one over an ambient space $N$ that is a germ around a compact analytic subset $C \subset N$ and let $E$ be a normal crossings divisor of $N$ all whose irreducible components are non-dicritical for $\mathcal{F}$. Assume that we have only pre-simple singularities, that is $\operatorname{Sing}^{*}(\mathcal{F}, E)=\emptyset$. Then there is a finite sequence of permissible blowings up

$$
N \stackrel{\pi_{1}}{\longleftarrow} N_{1} \stackrel{\pi_{2}}{\longleftarrow} N_{2} \ldots \stackrel{\pi_{k}}{\longleftarrow} N_{k}=N^{\prime}
$$


such that all the singularities of the transform $\mathcal{F}^{\prime}$ of $\mathcal{F}$ are simple singularities of $\mathcal{F}^{\prime}$ adapted to the divisor $E^{\prime}$ composed of the non-dicritical components of the total exceptional divisor in $N^{\prime}$.

The proof of this result is essentially based in the destruction of the resonances. In a quite easy way we first eliminate the pre-simple singularities of the type $\mathrm{C}$, getting a persistent situation of types A or B. Let us assume for simplicity that all our singularities are of type A (the strategy in the general case is very similar). In the two-dimensional case there is only one strategy for blowing up (to modify the non-simple points) but in higher dimension it is not evident what is the strategy to adopt. Let us look at the following example (due to P. Fortuny): take

$$
\underline{\lambda}=(-2,3 / 2+i, 3 / 2-i,-3) \text {. }
$$

If we adopt the (a priori reasonable) strategy of blowing up resonant centers of maximal dimension, we can take the center $x_{1}=x_{2}=x_{3}=0$ and after blowing up, in one of the charts we get

$$
\underline{\lambda}=(1,3 / 2+i, 3 / 2-i,-3) .
$$

Now, blowing up $x_{1}=x_{4}=0$ in one of the charts we get the initial situation

$$
\underline{\lambda}=(-2,3 / 2+i, 3 / 2-i,-3) .
$$

Thus, we have to be careful with the strategy to adopt. Actually, we can translate the problem of destruction of resonances to another known problem (toroidal embeddings) as follows. Given the resonant vector $\underline{\lambda} \in \mathbf{C}^{t}$ define the rational vector space

$$
\mathrm{L}(\underline{\lambda})=\left\{e=\left(e_{i}\right)_{i=1}^{t} \in \mathbf{Q}^{t} ; \sum_{i=1}^{t} e_{i} \lambda_{i}=0\right\} .
$$

The fact that $\underline{\lambda}$ is resonant means that $\mathrm{L}(\underline{\lambda}) \cap\left(\mathbf{Q}_{+}\right)^{t} \neq \emptyset$. Let $\mathrm{V}(\underline{\lambda}) \subset \mathbf{Q}^{t}$ be the orthogonal of $\mathrm{L}(\underline{\lambda})$. Then, for any non-null element $\underline{a} \in \mathrm{V}(\underline{\lambda}) \cap \mathbf{Z}^{t}$ we have a decomposition

$$
\underline{a}=\underline{p}-\underline{q}
$$

where $p, q \in \mathbf{Z}^{t}-\{0\}$. Consider the ideal generated by the binomials of the type

$$
\underline{x} \underline{\underline{p}}-\underline{x} \underline{q} .
$$

It defines a toric variety whose reduction of singularities gives the elimination of the resonances. Actually we can prove it directly in a globally coherent way: first we draw a characteristic polyhedra and second we use on it the global strategy derived from the Spivakovsky's solution to the weak Hironaka game [37].

7. Reduction of the singularities of foliations. Like in the two-dimensional case, we can establish the statement of reduction of the singularities of foliations concerning divisors in the ambient space that may have dicritical components. Let $\mathcal{F}$ be a singular foliation and $D$ a normal crossings divisor on the ambient space $N$. Denote by $E$ the union of the non-dicritical components of $D$ relatively to $\mathcal{F}$ and let $D_{c}$ be the union of the dicritical components.

Definition 17 . Take a point $P \in N$, we say that the foliation $\mathcal{F}$ and the divisor $D$ have normal crossings at the point $P$ if and only if the following conditions hold:

1. The point $P$ is either a non-singular point for $\mathcal{F}$ or a simple singularity for $\mathcal{F}$ adapted to $E$. 
2. Let $t$ be the dimensional type of $\mathcal{F}$ at the point $P$. Then there are local coordinates $\left(x_{1}, \ldots, x_{n}\right)$ at $P$ such that $\mathcal{F}$ is generated by a 1 -form $\omega$ of the type

$$
\omega=\sum_{i=1}^{t} a\left(x_{i}, \ldots, x_{t}\right) d x_{i}
$$

and $D_{c} \subset\left(x_{t+1} \ldots x_{n}=0\right)$ locally at $P$.

The complete result of reduction of singularities for a foliation should be the following

StATEment 18 (Reduction of singularities of a foliation). Let $\mathcal{F}$ be a singular foliation of codimension one over an ambient space $N$ of dimension $n$ that is a germ around a compact analytic subset $C \subset N$. Then there is a finite sequence of permissible blowings up

$$
N \stackrel{\pi_{1}}{\longleftarrow} N_{1} \stackrel{\pi_{2}}{\longleftarrow} N_{2} \ldots \stackrel{\pi_{k}}{\longleftarrow} N_{k}=N^{\prime}
$$

such that the transform $\mathcal{F}^{\prime}$ of $\mathcal{F}$ in $N^{\prime}$ and the total exceptional divisor $D^{\prime}$ have normal crossings everywhere.

This result is proved in dimensions two and three [8] and it is still a conjecture for $n \geq 4$. In dimension three, it is derived from the reduction to pre-simple singularities, after we get simple singularities and finally, we put by means of additional blowings up the dicritical components of the divisor having normal crossings with the foliation and the non-dicritical divisor. The last step is surely true and not too difficult in any dimension and a more general context (modulo the use of one of the known algorithms [2] for the reduction of singularities of hypersurfaces) that we call the conditionated reduction:

Statement 19 (Conditionated reduction). Let $\mathcal{F}$ be a singular foliation of codimension one over an ambient space $N$ of dimension $n$ that is a germ around a compact analytic subset $C \subset N$. Consider a normal crossings divisor $E$ on $N$ which is non-dicritical for $\mathcal{F}$ and let $S$ be a hypersurface on $N$ (may be singular, with several irreducible components). Assume that all the singularities of $\mathcal{F}$ are simple singularities adapted to $E$. Then, there is a finite sequence of permissible (in an enlarged sense) blowings up

$$
N \stackrel{\pi_{1}}{\longleftarrow} N_{1} \stackrel{\pi_{2}}{\longleftarrow} N_{2} \ldots \stackrel{\pi_{k}}{\longleftarrow} N_{k}=N^{\prime}
$$

such that the transforms $\mathcal{F}^{\prime}$ of $\mathcal{F}$ and $D^{\prime}$ of $E \cup S$ have normal crossings everywhere.

8. Thom's conjecture on the existence of integral hypersurfaces. One of the applications that we would like to present here of the known results of reduction of singularities of the foliations is the proof of the existence of an integral hypersurface for any germ of non-dicritical holomorphic foliation $\mathcal{F}$ over $\left(\mathbf{C}^{n}, 0\right)$.

Before starting with the proof itself, let us look at the non-dicriticalness condition, that we have studied in $[5,6]$. The more precise definition for a non-dicritical foliation is

Definition 20. We say that $\mathcal{F}$ is non-dicritical if and only if for any finite sequence of permissible blowings up

$$
\left(\mathbf{C}^{n}, 0\right)=N \stackrel{\pi_{1}}{\longleftarrow} N_{1} \stackrel{\pi_{2}}{\longleftarrow} N_{2} \ldots \stackrel{\pi_{k}}{\longleftarrow} N_{k}=N^{\prime}
$$

all the irreducible components of the total exceptional divisor $E^{\prime}$ are non-dicritical components for the transform $\mathcal{F}^{\prime}$ of $\mathcal{F}$. 
In dimension two, to be dicritical is equivalent to have infinitely many integral curves. This is directly done by the reduction of the singularities. The dicriticalness in dimension two can be tested with the canonical reduction of the singularities (actually, assuming that the reduction of singularities exists in dimension $n$, we think that any reduction of the singularities is enough to test the non-dicriticalness condition). Now, appearing a dicritical component in the two-dimensional reduction of the singularities gives infinitely many integral curves, by projecting the integral curve passing though each non-singular point (not a corner) of this dicritical component. Conversely, if we have infinitely many integral curves, there is necessarily one dicritical component in the reduction of the singularities. More precisely, for a non-dicritical foliation in dimension two we have a bijection

$$
\left\{\begin{array}{c}
\text { formal integral } \\
\text { curves of } \mathcal{F}
\end{array}\right\} \leftrightarrow\left\{\begin{array}{c}
\text { singular trace points } \\
\text { after reduction of singularities }
\end{array}\right\}
$$

and the correspondence gives convergent integral curves if and only if the corresponding trace simple singularity has convergent integral curve transverse to the divisor.

A particular corollary of the above discussion is that in dimension two and the dicritical case we always have at least one convergent integral curve (actually infinitely many of them). This is not the situation in higher dimension. The reason is that a germ of hypersurface in a point obtained after some blowings up does not necessarily project in a hypersurface in the initial ambient space, we need to have a coherent global object. Look at the following example of Darboux studied by Jouanolou [25]. Consider the germ of foliation on $\left(\mathbf{C}^{3}, 0\right)$ given by the integrable homogeneous 1 -form

$$
\omega=\left(x^{m} y-z^{m+1}\right) d x+\left(y^{m} z-x^{m+1}\right) d y+\left(z^{m} x-y^{m+1}\right) d z
$$

$(m \geq 2)$. It is dicritical (actually radially dicritical). After one blowing up of the origin, the exceptional divisor is a projective plane $\mathbf{P}^{2}$ transverse to the transform of our foliation. In fact the foliation is a foliation by cones and the leaves over the projective plane are the projectivization of that cones. It can be proved [25] that this foliation over $\mathbf{P}^{2}$ has no integral algebraic curve. Hence the initial foliation has no integral hypersurface, since otherwise it is a cone whose projectivization is a closed integral algebraic curve of the foliation induced over $\mathbf{P}^{2}$.

Thus, it is reasonable to consider Thom's question only in the non-dicritical case. Assume in the sequel that the foliation $\mathcal{F}$ is non-dicritical. We will give an outline of the proof of the following theorem, done for dimension two in [4], for dimension three in [9] and for dimension bigger than three - in [11].

THEOREM 21 (Existence of convergent integral hypersurface). Let $\mathcal{F}$ be a non-dicritical foliation over $\left(\mathbf{C}^{n}, 0\right)$. Then there is at least one germ of convergent integral hypersurface for $\mathcal{F}$.

In dimension two, the first proof of the existence of a convergent integral curve for $\mathcal{F}$ is due to Camacho and Sad [4]. It uses first the reduction of the singularities of $\mathcal{F}$ and second an invariant of global-local nature (of residue type) that allows to find a contradiction with the fact that there is no convergent integral curve. This proof is existential and involves a complicated combinatorial argument. Other direct proofs that construct step by step the integral curve are in [13] or in [12] where the Newton-Puiseux method is extended to the case of differential equations.

In dimension three, the existence of convergent integral hypersurface is a consequence of the existence of reduction of singularities and the description of the simple singularities 
that we obtain [9]. Denote by

$$
\pi: N^{\prime} \rightarrow N=\left(\mathbf{C}^{3}, 0\right)
$$

a non-dicritical reduction of the singularities of the foliation $\mathcal{F}$. Let $\mathcal{F}^{\prime}$ be the transform of $\mathcal{F}$ and $E^{\prime}$ the total exceptional divisor. Thus $E^{\prime}$ is a normal crossings divisor on $N^{\prime}$, all its components are non-dicritical for $\mathcal{F}^{\prime}$ and any singular point $P \in \operatorname{Sing} \mathcal{F}^{\prime}$ is a simple singularity for $\mathcal{F}$ adapted to $E^{\prime}$. Recall that we have two types of singularities:

Corner singularities. The only integral hypersurfaces through a corner singularity are the irreducible components of the divisor $E^{\prime}$. The singularities near a corner singularity are also corner singularities.

Trace singularities. There is exactly one integral hypersurface $\hat{H}$ through a trace singularity different from the irreducible components of the divisor. Moreover this hypersurface is transversely formal (and not merely formal) and the trace singularities near a trace singularity correspond exactly to $E^{\prime} \cap \hat{H}$.

Thus, the trace singularities form a closed analytic subset of $N^{\prime}$. Denote it by $\operatorname{Tr}\left(\mathcal{F}^{\prime}, E^{\prime}\right)$. Each irreducible component of it is non-singular of codimension two and $\operatorname{Tr}\left(\mathcal{F}^{\prime}, E^{\prime}\right)$ has normal crossings with $E^{\prime}$. Denote by $\hat{N}^{\prime}$ the formal analytic space obtained from $N^{\prime}$ along the exceptional divisor $E^{\prime}$. The nature of that formal integral hypersurfaces $\hat{H}$ allows to glue them over each connected component of the $\operatorname{set} \operatorname{Tr}\left(\mathcal{F}^{\prime}, E^{\prime}\right)$. We get so a closed hypersurface $\tilde{H}$ of $\hat{N}^{\prime}$ that is an integral hypersurface of the foliation $\hat{\mathcal{F}}^{\prime}$ induced by $\mathcal{F}$ in $\hat{N}^{\prime}$. Moreover, the hypersurfaces obtained in this way are the only integral hypersurfaces of $\hat{\mathcal{F}}^{\prime}$ different from the irreducible components of $E^{\prime}$. Thus, considering the morphism

$$
\hat{\pi}: \hat{N}^{\prime} \rightarrow \hat{N}=\left(\mathbf{C}^{\hat{3}}, 0\right)
$$

induced by the proper morphism $\pi$, we get the following bijections:

$$
\left.\begin{array}{c}
\text { Formal integral } \\
\text { hypersurfaces of } \mathcal{F}
\end{array}\right\} \leftrightarrow\left\{\begin{array}{c}
\text { Integral hypersurfaces } \\
\text { of } \hat{\mathcal{F}}^{\prime} \text { in } \hat{N}^{\prime}
\end{array}\right\} \leftrightarrow\left\{\begin{array}{c}
\text { Connected components } \\
\text { of } \operatorname{Tr}\left(\mathcal{F}^{\prime}, E^{\prime}\right)
\end{array}\right.
$$

Moreover, in this correspondence we get a convergent integral hypersurface of $\mathcal{F}$ if and only if the corresponding connected component $T$ of $\operatorname{Tr}\left(\mathcal{F}^{\prime}, E^{\prime}\right)$ supports a convergent integral hypersurface $\hat{H}$ (equivalently, the corresponding $\tilde{H}$ comes from a convergent hypersurface in $N^{\prime}$ ). This property can be tested in any point of the connected component $T$ : the easiest one will be a generic point of dimensional type two. Thus we are allowed to prove that there is at least one of such points. This will be done by using the corresponding result in dimension two. By a classical transversality result [32] we can consider a non-singular two-dimensional subspace

$$
\Delta \subset\left(\mathbf{C}^{3}, 0\right)
$$

such that if $\omega$ generates $\mathcal{F}$ then the restriction $\bar{\omega}$ of $\omega$ to $\Delta$ has only isolated singularity. By the result in dimension two, there is at least one convergent integral curve $\Gamma$ for $\bar{\omega}$. Let $\Gamma^{\prime}$ be the strict transform of $\Gamma$ by the morphism $\pi$ (note that all the centers in $\pi$ are contained in the singular locus of the foliation and thus they do not contain $\Gamma$ ). There is a point $P^{\prime} \in \Gamma^{\prime} \cap E^{\prime}$. This point is necessarily a trace singularity that supports a convergent $\hat{H}$. To see this, let us assume that up to additional blowings up of points, the strict transform $\Gamma^{\prime}$ is non-singular at $P^{\prime}$ and has normal crossings with $E^{\prime}$, in particular there is only an irreducible component of $E^{\prime}$ through $P^{\prime}$. Now $\hat{H}$ is locally an analytic cylinder over $\Gamma^{\prime}$ directed by the singular locus Sing $\mathcal{F}$, that is non-singular and of codimension two. Hence 
$\hat{H}$ is convergent and thus the corresponding integral hypersurface of $\mathcal{F}$ is so. This ends the proof of the result in the three-dimensional case.

Now let us give the guides to the proof in higher dimensions done in [11]. It uses the result in dimension three, the generic equireduction and a cohomological triviality that works for the passage 3 to $n$, but not 2 to $n$. Let us denote by $B \subset\left(\mathbf{C}^{n}, 0\right)$ the set of bad points of $\mathcal{F}$, that is we have generic equireduction over any singular point not in $B$. The set $B$ is a closed analytic set of codimension at least three. Invoking the above transversality theorem, we can consider a three-dimensional non-singular subspace

$$
M=\left(\mathbf{C}^{3}, 0\right) \subset\left(\mathbf{C}^{n}, 0\right)
$$

such that the restriction $\bar{\omega}$ to $M$ of a generator $\omega$ has singular locus of codimension at least two and moreover $M \cap B$ is an isolated point. Denote by $\overline{\mathcal{F}}$ the restriction to $M$ of $\mathcal{F}$. We know that $\overline{\mathcal{F}}$ is non-dicritical and thus, by application of the result in dimension three, there is one convergent integral hypersurface $\bar{H} \subset M$ of $\overline{\mathcal{F}}$. Consider a small open annulus $\mathcal{C} \subset M=\left(\mathbf{C}^{3}, 0\right)$ and the open set

$$
W=\mathcal{C} \times\left(\mathbf{C}^{n-3}, 0\right) \subset\left(\mathbf{C}^{n}, 0\right) .
$$

Locally at each point $P$ of $\bar{H} \cap W$ the integral hypersurface $\bar{H}$ of $\overline{\mathcal{F}}$ extends in a unique way to a germ at $P$ of integral hypersurface $H_{P}$ for $\mathcal{F}$. This is evident if the point $P$ is a nonsingular point of $\mathcal{F}$. If $P \in \operatorname{Sing} \mathcal{F}$ we use the fact that we have a canonical equireduction; the given $\bar{H}$ contains a germ of curve at $P$ not in the singular locus that points exactly one of the germs of integral hypersurfaces that we get by generic equireduction. Recall that the generic equireduction in the non-dicritical case is like a two-dimensional reduction with parameters given by the singular locus. Glueing together the germs $H_{P}$ we obtain a closed hypersurface $\tilde{H}$ that is an integral hypersurface of the restriction of $\mathcal{F}$ to $W$. Note also that $\bar{H} \cap W \subset \tilde{H}$. Now applying classical results of cohomological triviality [36] we know that $\tilde{H}$ extends to a closed hypersurface $H$ of $\left(\mathbf{C}^{n}, 0\right)$. Obviously $H$ is an integral hypersurface of $\mathcal{F}$ and $\bar{H} \subset H$, in particular the origin is in $H$ and it is a well defined germ. This ends the proof.

9. The frontier of a pfaffian hypersurface. In the real analytic case we can use the above results of reduction of singularities to give a description of the frontier of a pfaffian hypersurface [10]. One situation is to be excluded: the spiraling situation. The idea is to show that outside this spiraling phenomena, the frontier of a pfaffian hypersurface can be constructed as a finite union of proper projections of pfaffian hypersurfaces, in the same spirit as the theory of subanalytic sets [23, 24, 28, 29].

The elementary blocks that we use are the so called pfaffian hypersufaces with Rolle property (see [26], for another approach to this objects). A pfaffian hypersurface is a 4-uple

$$
\mathcal{H}=(V, M, N ; \mathcal{F})
$$

where $N$ is a real analytic ambient space and $\mathcal{F}$ a singular foliation of codimension one over $N$ (we always work with objects that admit a good complexification). The subset $M \subset N$ is an open semianalytic subset of $N$ such that $N \cap \operatorname{Sing} \mathcal{F}=\emptyset$. Finally, the set $V$, that we call the support of the pfaffian hypersurface, is a leaf of the non-singular foliation induced by $\mathcal{F}$ on $M$. In this definition we admit the degenerate case $\mathcal{F} \equiv 0$; then the support should be a connected component of the semianalytic open set $M$. 
We say that $\mathcal{H}$ has the Rolle property if and only if any analytic parametrized curve $\gamma$ in $M$ transverse to $\mathcal{F}$ cuts the leaf $V$ at most once. The spiraling situation is an example of non-Rolle property (it is exactly the contrary under certain assumptions [34]). Actually, when the semianalytic set $M$ is simply connected we always have the Rolle property [34]. Thus, making a locally finite decomposition of $M$ as union of simply connected semianalytic open sets, we can express any pfaffian hypersurface in a reasonable way as a, possible infinite, union of pfaffian rollian hypersurfaces.

The objective is to describe the frontier of a given rollian pfaffian hypersurface. In [10] we prove the following theorem.

THEOREM 22. Let $\mathcal{F}$ be a singular foliation over $N$. Given a rollian pfaffian hypersurface $\mathcal{H}=(V, M, N ; \mathcal{F})$, the frontier $\partial V$ is an $\mathcal{F}$-set. More generally, the topological closure $\bar{W}$ of any $\mathcal{F}$-set $W$ is also an $\mathcal{F}$-set.

Of course, the meaning of the above result is contained in the definition of $\mathcal{F}$-set. By definition, an $\mathcal{F}$-set is a locally finite union of elementary $\mathcal{F}$-sets. Thus, let us define what is an elementary $\mathcal{F}$-set.

An admissible morphism $\Delta=\left(\delta, \alpha=\left\{\alpha_{i}\right\}_{i=1}^{k}\right)$ is given by a finite sequence of morphisms:

$$
N=N_{0} \stackrel{\alpha_{1}}{\longleftarrow} N_{1} \stackrel{\alpha_{2}}{\longleftarrow} N_{2} \ldots \stackrel{\alpha_{k}}{\longleftarrow} N_{k}=N^{\prime}
$$

such that each $\alpha_{i}$ is either a local blowing up or a closed immersion. Then we put

$$
\delta=\alpha_{k} \circ \ldots \circ \alpha_{1}: N^{\prime} \longrightarrow N \text {. }
$$

Denote by $\mathcal{F}_{i}$ the transform of $\mathcal{F}_{i-1}$ by $\alpha_{i}$, where $\mathcal{F}_{0}=\mathcal{F}$. We say that $\Delta^{*} \mathcal{F}=\mathcal{F}_{k}$ is the transform of $\mathcal{F}$ by the admissible morphism $\Delta$. It is very important to remark that $\Delta^{*} \mathcal{F}$ is not necessarily the transform $\delta^{-1} \mathcal{F}$ of $\mathcal{F}$ by $\delta$. The fact that we construct the transforms in an iterative way is closely related with the dicriticalness property. As we shall see later, it explains also geometrically some of the transcendence properties of the frontier of the pfaffian hypersurfaces and more generally of the $\mathcal{F}$-sets. Look at the following example. Take the radial foliation in dimension two given in $\mathbf{R}^{2}$ by

$$
\omega=y d x-x d y \text {. }
$$

Let $\alpha_{1}: N_{1} \rightarrow \mathbf{R}^{2}$ be the blowing up of the origin and let $\alpha_{2}: E=N_{2} \rightarrow N_{1}$ be the closed immersion of the exceptional divisor $E$ in $N_{1}$. Put $\delta=\alpha_{1} \circ \alpha_{2}$. Then $\delta^{-1} \mathcal{F} \equiv 0$ but $\Delta^{*} \mathcal{F} \not \equiv 0$, since the first blowing up is dicritical.

Definition 23. A subset $W \subset N$ is an elementary $\mathcal{F}$-set if and only if $W$ is of the form $W=\delta(V)$ for an admissible morphism $\Delta=\left(\delta, \alpha=\left\{\alpha_{i}\right\}_{i=1}^{k}\right)$ and a rollian pfaffian hypersurface

$$
\mathcal{H}=\left(V, M, N_{k} ; \Delta^{*} \mathcal{F}\right)
$$

such that $\delta: V \rightarrow N$ is a proper mapping.

It is obvious from the definition that a rollian pfaffian hypersurface on $N$ is an $\mathcal{F}$-set.

A basic argument, even in order to derive very elementary properties of the $\mathcal{F}$-sets is the finiteness property that is proved in [33]:

The intersection of a locally finite number of pfaffian rollian hypersurfaces and semianalytic sets is also locally finite. 
The proof of this result is based on the existence of stratifications simultaneously compatible with the foliations and the semianalytic sets. This is an argument that we will also use later.

By using the finiteness property and results of rectilinearization of Hironaka as well as stratifications in the spirit of ojasiewicz, we get a list of elementary properties for the $\mathcal{F}$-sets:

1. The class of $\mathcal{F}$-sets is closed for locally finite union.

2. From the connectedness viewpoint, each $\mathcal{F}$-set $W$ is arc connected, has a finite (locally finite) number of connected components and each connected component of $W$ is also an $\mathcal{F}$-set.

3. The $\mathcal{F}$-sets are locally defined in the sense that a subset of $N$ is an $\mathcal{F}$-set iff it is so in restriction to a basis of open sets.

4. The intersection of an $\mathcal{F}$-set and a subanalytic subset of $N$ is also an $\mathcal{F}$-set. But it is not clear that the intersection of two $\mathcal{F}$ - sets will be an $\mathcal{F}$-set (actually we think that this is not true).

5. An $\mathcal{F}$-set is a finite union of $\mathcal{F}$-sets that are non-singular subvarieties of the ambient space $N$. Unfortunately we do not get a complete stratification.

The structure of the proof of the theorem above is as follows. By a Whitney's type wing lemma [27] we reduce ourselves to the exterior of an arbitrary subset of codimension three. Outside this bad set, we can use generic equireduction that gives to us the result. Let us detail a little bit more the argument.

First, we can work by induction over the ambient space. Second, the local description of the $\mathcal{F}$-sets and the rectilinearization of Hironaka [23] reduce the problem to the study of a pfaffian hypersurface $V$ on a quadrant $M$ (the frontier of $M$ will be a normal crossings divisor). Up to invoke the induction over the dimension and the wing lemma, we can assume that we have generic equireduction over the singular locus. Now, we do the equireduction. Along this blowing up procedure our total transform divisor (the new frontier of $M$ ) has dicritical and non-dicritical components. In the case of dicritical components, we get a piece of the frontier that corresponds to the same problem in lower dimension (the new ambient space will be the divisor). Along the non-dicritical components we get a quite trivial (subanalytic) part of the frontier looking how a leaf of a simple singularity accumulates over the divisor. Actually, the presence of the dicritical components corresponds to a certain transcendence of the frontier.

Let us end this paragraph with an example [10] in dimension 4 (the first non-trivial one) that illustrates the behavior cited above. Consider the foliation $\mathcal{F}$ on $\mathbf{R}^{4}$ generated by

$$
\left.\omega=2 x t z^{2}(y d x-x d y)+y^{3}\left(t d z-z^{2} d t\right)\right)
$$

and let $V$ be any leaf over the semianalytic open set $M=\{y>0, z>0, t>0\}$. The analytic function $f$ defined on $M$ by

$$
f(x, y, z, t)=(x / y)^{2}-1 / z-\log t
$$

is a first integral of the foliation over $M$. Thus assume $V=(f=0)$. Let us study the closure $\bar{V}$ of $V$ along the 2-plane $x=y=0$ (the most interesting piece). Doing the blowing up with this center, in a chart $x=v y$ the transformed foliation is given by

$$
\omega^{\prime}=2 v t z^{2} d z+t d z-z^{2} d t
$$


the exceptional divisor $y=0$ is dicritical and we have a the first integral given in the inverse image of $M$ by

$$
f^{\prime}=v^{2}-1 / z-\log t
$$

(tangent to the fibers of the projection $(v, y, z, t) \rightarrow(v, z, t)$ over the exceptional divisor). Thus the closure over the exceptional divisor corresponds to the closure of

$$
v^{2}-1 / z-\log t=0, z>0, t>0 \text {. }
$$

Projecting over the two plane $x=y=0$ we get

$$
\{t \geq \exp (-1 / z), z>0\} \cup\{t \geq 0, z=0\}
$$

which is not subanalytic.

\section{References}

[1] J. M. Aroca, H. Hironaka, J. L. Vicente, The Theory of the Maximal Contact, Memorias de Matemática del Instituto "Jorge Juan" 29, Madrid, 1975.

[2] J. M. Aroca, H. Hironaka, J. L. Vicente, Desingularization Theorems, Memorias de Matemática del Instituto "Jorge Juan" 30, Madrid, 1975.

[3] C. A. Briot, J. C. Bouquet, Propriétés des fonctions définies par les équations différentielles, Journal de l'École Polytechnique 36 (1856), 133-198.

[4] C. Camacho, P. Sad, Invariant varieties through singularities of holomorphic vector fields, Ann. of Math. (2) 115 (1982), 579-595.

[5] F. Cano, Dicriticalness of a singular foliation, in: Holomorphic Dynamics (México 1986), X. Gómez-Mont, J. Seade, A. Verjovski (eds.), Lecture Notes in Math. 1345, Springer, Berlin, 1988, 73-94.

[6] F. Cano, Foliaciones singulares dicríticas, Mem. Real Acad. Cienc. Exact. Fís. Natur. Madrid 24 (1989)

[7] F. Cano, Reduction of the singularities of non-dicritical singular foliations. Dimension three, Amer. J. Math. 115 (1993), 509-588.

[8] F. Cano, Reduction of singularities of codimension one foliations in dimension three, in preparation, Univ. Valladolid.

[9] F. Cano, D. Cerveau, Desingularization of non-dicritical holomorphic foliations and existence of separatrices, Acta Math. 169 (1992), 1-103.

[10] F. Cano, J. M. Lion, R. Moussu, Frontière d'une hypersurface pfaffienne, Ann. Sci. École Norm. Sup. (4) 28 (1995), 591-646.

[11] F. Cano, J. F. Mattei, Hypersurfaces intégrales des feuilletages holomorphes, Ann. Inst. Fourier (Grenoble) 42 (1992), 49-72.

[12] J. Cano, An extension of the Newton-Puiseux polygon construction to give solutions of pfaffian forms, Ann. Inst. Fourier (Grenoble) 43 (1993), 125-142.

[13] J. Cano, Construction of invariant curves for holomorphic vector fields, Proc. Amer. Math. Soc. 125 (1997), 2649-2650.

[14] V. Cossart, Forme normale pour une fonction sur une variété de dimension trois en caractéristique positive, Thèse d'Etat, Orsay, 1988.

[15] J. Giraud, Forme normale d'une fonction sur une surface de caractéristique positive, Bull. Soc. Math. France 111 (1983), 109-124.

[16] J. Giraud, Condition de Jung pour les revêtements radiciels de hauteur un, in: Algebraic Geometry (Tokyo/Kyoto 1982), M. Raynaud and T. Shioda (eds.), Lecture Notes in Math. 1016, Springer, Berlin, 1983, 313-333. 
[17] H. Hironaka, Resolution of singularities of an algebraic variety over a field of characterictic zero, Ann. of Math. (2) 79 (1964), 109-203, 205-326.

[18] H. Hironaka, Introduction to the Theory of Infinitely Near Singular Points, Memorias de Matemática del Instituto "Jorge Juan" 28, Madrid, 1975.

[19] H. Hironaka, La voûte étoilée, Astérisque 7-8 (1973), 13-20.

[20] H. Hironaka, Idealistic exponents of singularity, in: Algebraic Geometry, John Hopkins Univ. Press, Baltimore, 1977, 52-125.

[21] H. Hironaka, Characteristic polyhedra of singularities, J. Math. Kyoto Univ. 7 (1967), 251-293.

[22] H. Hironaka, Desingularization of excellent surfaces, appendix in: V. Cossart, J. Giraud, U. Orbanz, Resolution of Surfaces, Lecture Notes in Math. 1101, Springer, Berlin, 1984.

[23] H. Hironaka, Introduction to Real-analytic Sets and Real-analytic Maps, Istituto Matematico L. Tonelli, Pisa, 1973.

[24] H. Hironaka, Subanalytic sets, in: Number Theory, Algebraic Geometry and Commutative Algebra, Kinokuniya, Tokyo, 1973, 453-493.

[25] J. P. Jouanolou, Équations de Pfaff algébriques, Lecture Notes in Math. 708, Springer, Berlin, 1979.

[26] A. G. Khovanskii, Real analytic varieties with the finiteness property and complex abelian integrals, Functional Anal. Appl. 18 (1984), 119-127.

[27] J. M. Lion, Un lemme d'aile pour les ensembles pfaffiens, C. R. Acad. Sci. Paris Sér. I Math. 316 (1993), 187-189.

[28] S. Łojasiewicz, Ensembles semi-analytiques, Inst. de Hautes Études Scientifiques, Buressur-Yvette, 1965.

[29] S. Łojasiewicz, M. A. Zurro, Una introducción a la geometría semi y sub analítica, Secretariado de Publicaciones, Universidad de Valladolid D.L., ed. Serie Ciencias 6, 1993.

[30] B. Malgrange, Frobenius avec singularités. I. Codimension un, Inst. Hautes Études Sci. Publ. Math. 46 (1976), 162-173.

[31] J. Martinet, Normalisation des champs de vecteurs holomorphes (d'après A.-D. Bryuno), in: Séminaire Bourbaki, vol. 1980/81, Lecture Notes in Math. 901, Springer, Berlin, 1982, exp. 564, 55-70.

[32] J. F. Mattei, R. Moussu, Holonomie et intégrales premières, Ann. Sci. École Norm. Sup. (4) 13 (1980), 469-523.

[33] R. Moussu, C. Roche, Théorie de Hovanskii et problème de Dulac, Invent. Math. 105 (1991), 431-441.

[34] R. Moussu, C. Roche, Théorèmes de finitude pour les variétés pfaffiennes, Ann. Inst. Fourier (Grenoble) 42 (1992), 393-420.

[35] A. Seidenberg, Reduction of the singularities of the differential equation $A d y=B d x$, Amer. J. Math. 90 (1968), 248-269.

[36] Y. T. Siu, Techniques of Extension of Analytic Objects, Lecture Notes in Pure and Appl. Math. 8, Dekker, New York, 1974.

[37] M. Spivakovsky, A solution to Hironaka's polyhedra game, in: Arithmetic and Geometry vol. II, M. Artin and J. Tate (eds.), Progr. Math. 36, Birkhäuser, Boston, 1983, 419-432.

[38] M. Spivakovsky, Resolution of singularities, preprint, Grenoble-Valladolid-Toronto, 1996. 